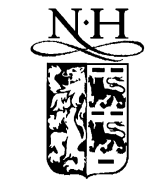

ELSEVIER

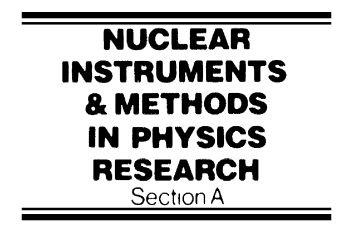

www.elsevier.nl/locate/nima

\title{
A technique for the absolute measurement of the $W$-value for X-rays in counting gases
}

\author{
F.L.R. Vinagre*, C.A.N. Conde \\ Departamento de Física, FCTUC, Universidade de Coimbra, P-3004-516 Coimbra, Portugal
}

Received 6 August 1999; received in revised form 13 January 2000; accepted 9 February 2000

\begin{abstract}
A technique was developed for the absolute measurement of the $W$-value (the mean energy for the production of an electron-ion pair) for low-energy X-rays in a wide range of gases at atmospheric pressures, with a standard uncertainty better than $1 \%$. This technique is based on the absolute measurement of the primary ionization charge produced by $\mathrm{X}$-ray photons from a constant intensity monoenergetic X-ray source, e.g. a long lifetime radioactive source. The ionization charge is calibrated by the number of X-ray photons absorbed in the gas, counted with a photon detector. For this purpose, a hybrid detector system was tested and its use in $W$-value measurements was investigated. The technique was applied to pure xenon at 825 Torr with $5.9 \mathrm{keV} \mathrm{X-rays} \mathrm{and} \mathrm{a} W$-value of $21.61_{-0.10}^{+0.14} \mathrm{eV}$ was obtained for a $68 \%$ confidence level. The required corrections and the different factors contributing to the accuracy of the results are discussed. The advantages and limitations of this technique are explored and future developments are discussed. (C) 2000 Elsevier Science B.V. All rights reserved.
\end{abstract}

Keywords: $W$-value; Ionization yield; Xenon; Radiation physics; Dosimetry; X-ray detectors

\section{Introduction}

The microscopic action of an ionizing radiation in a medium is of great interest in dosimetry/microdosimetry and radiation physics. The so-called $W$-value, the energy required to form a primary electron, is usually used to characterize the process of complete energy dissipation of the radiation in the medium. The $W$-value is the ratio of the incident radiation energy $E$ to the mean number $\bar{N}$ of primary electrons (the ionization yield) produced in the gas after the complete dissipation of $E$ [1], i.e.

$W=E / \bar{N}$.

\footnotetext{
*Corresponding author. Tel.: + + 351-239-410667; fax: $++351-239-829158$.

E-mail address: fleonor@saturno.fis.uc.pt (F.L.R. Vinagre).
}

Most of the experimental $W$-values published in the literature were measured with relative methods that rely on the previous knowledge of the absolute $W$-value for a reference gas. These methods are based on the comparison between the ionization yields in the two gases, either through the measurement of ionization currents or charges [2,3], pulse amplitudes [4-6] or counting rates in fixed ionization current systems $[7,8]$.

For low-energy X-rays, which deposit a small amount of energy in a medium, the ionization yield is difficult to measure experimentally. Several $W$ value absolute experimental methods have been developed for this purpose. Lyons et al. [9] used an ionization chamber to collect the primary ionization current together with a proportional counter and a $\mathrm{Si}(\mathrm{Li})$ detector for the determination of the 
absorbed energy, and achieved an accuracy of $\pm 2 \%$. Srdoc [10] developed a method that calibrates the X-ray pulse amplitude from the amplitude distribution of a proportional counter (PC) with the single electron pulse amplitude. This method is restricted to gases with a sufficient amount of a quenching gas, for which the PC works in the proportional region at high gains [11]. Yet, such high gains cannot always be achieved for all the gases of interest in $W$-value measurements, as is the case of most of the pure gases.

Recently, Pansky et al. [12,13] developed a method based on the technique of individually counting the primary electrons $N$ produced by a single X-ray photon in a low-pressure gas, combining experimental work with Monte Carlo simulation of the detection process. This method allowed a variable accuracy up to $\pm 3 \%$ and it is restricted to very low X-ray energies (110-1486 eV) and gases at rather low pressures (about 10 Torr). At such low pressures, the $W$-value may be different from its value at the pressures normally used for X-ray dosimetry and spectrometry (around 1000 Torr), since different collisional processes might be involved in the production of primary electrons. Also, since the $W$-value is energy dependent as a result of atomic shell effects $[14,15]$, the results cannot be extrapolated to larger energies.

Our group, in Coimbra, developed an absolute method [16], restricted to scintillating gases, with accuracies of about \pm 2 to $\pm 3 \%$, which is the point of departure of the present work.

\section{Experimental system}

The method used is based on the absolute measurement of the mean primary electron charge, $Q$, produced by the complete absorption of a large number $n$ of monoenergetic X-ray photons (each with energy $E$ ). To obtain the $W$-value, both $Q$ and $n$ need to be measured experimentally. To do so, the method developed involves two sequential stages (interchangeable): one to measure the primary electron charge and another to measure the overall energy absorbed, as in our previous work [16].

A hybrid detector system was constructed to merge both these stages. The system, see Fig. 1, was designed

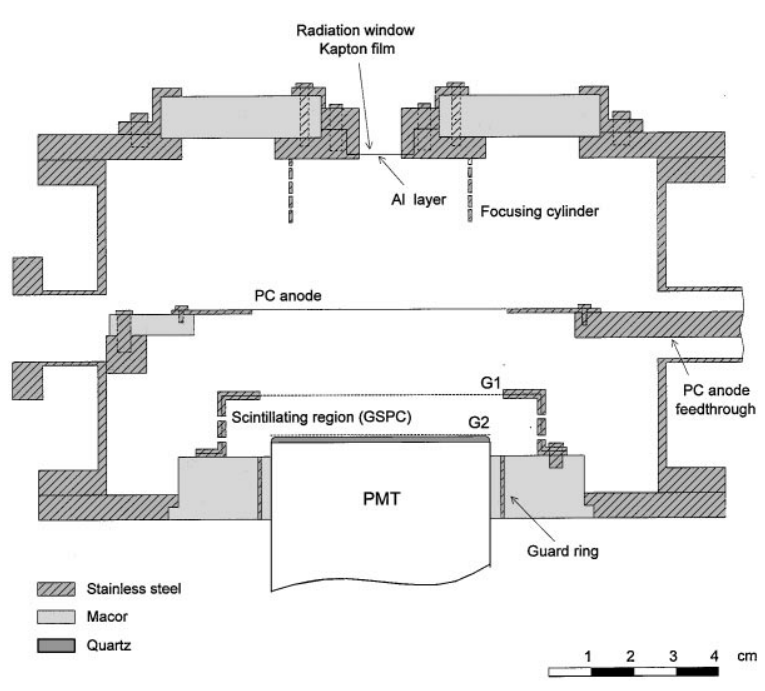

Fig. 1. Cross section of the hybrid detector system constructed.

to combine an ionization chamber (IC), a proportional counter (PC) and a gas proportional scintillation counter (GPSC). The X-ray entrance window is made of $25 \mu \mathrm{m}$ thick Kapton film. In its inner surface, an aluminum film ( $<200 \mathrm{~nm}$ thick) was vacuum deposited to provide electrical conductivity.

In the first stage, we use the experimental system working as a total absorption IC for the measurement of the ionization charge. In this mode, the detector has a $6 \mathrm{~cm}$ long absorption/drift region between the radiation entrance window and grid G1 (a stainless steel circular mesh). The electrons and positive ions created by the incident radiation drift towards opposite electrodes (at fixed potentials) inducing a charge on the grid G1, at the virtual ground potential. This charge is transferred to an electrometer (Keithley model 6512 programmable electrometer), directly connected to grid G1 through a highly insulating feedthrough that crosses the detector body (not shown in Fig. 1). Grid G2 is at a slightly repulsive and constant potential to repel the primary electrons, during the first stage. In the absence of charge multiplication, the overall induced charge produced by $n \mathrm{X}$-ray photons is $Q=n \bar{N} e$, where $e$ is the electron charge. To account for the ionization charge due to background radiation and the residual leakage currents, the background charge $Q_{\mathrm{b}}$ is obtained in the absence of 
the radiation source, in a prior measurement. With the X-ray source placed in a fixed position the ionization plus background charge, $Q+Q_{\mathrm{b}}$, is read.

In the second stage, the system acts as a photon detector to count the number $n$ of absorbed X-ray photons in the gas. Therewith one can directly obtain the overall absorbed energy $n E$ in the gas. The radiation source is kept exactly in the same position as during the first stage. The number of pulses in the photon detector (either a PC or a GPSC) is counted with an electronic counter (EC) and their amplitude distribution measured with a multichannel analyzer (MCA). When operating as a PC, the primary electron cloud drifts towards the anode wire, facing an increasing electric field and standard PC operation takes place [1]. PC pulses are extracted from the gold-plated $50 \mu \mathrm{m}$ diameter tungsten anode wire, located $4 \mathrm{~cm}$ from the entrance window and $2 \mathrm{~cm}$ from grid G1. On the other hand, when operating as a GPSC, the primary electrons created drift through the absorption/drift region between the entrance window and a planar grid $\mathrm{G} 1$ into a $1 \mathrm{~cm}$ deep scintillation region that extends up to grid G2. Here they produce a scintillating signal that is detected by a photomultiplier tube (PMT) THORN EMI model 9266QB with a quartz window. G2 is a chromium mesh deposited by vacuum evaporation onto the photomultiplier high-purity quartz window. A detailed description of a standard GPSC operation can be found elsewhere [17]. Finally, the number $n_{\mathrm{b}}$ of counts due to spectral electronic noise and background radiation (mainly cosmic radiation) is obtained after removing the X-ray source.

The $W$-value is then given by

$W=n E e / Q$.

This new technique yields an improved accuracy relatively to the earlier one [16] as a result of a series of technical improvements in the detector system design, the gas handling and purification system and the interface between the detector and electrometer. Moreover, the use of both the GPSC and the PC modes in the second stage allows studying scintillating and non-scintillating gases with this system, allowing also the cross-checking of the method with scintillating gases.

\section{Results and discussion}

For the present measurements, we used a $10 \mathrm{mCi}$ ${ }^{55} \mathrm{Fe}$ radioactive source, which emits the $\mathrm{Mn}$ $\mathrm{K}_{\alpha}$ and $\mathrm{K}_{\beta}$ fluorescence $\mathrm{X}$-ray lines with energies of 5.895 and $6.492 \mathrm{keV}$, respectively. A chromium filter (a $0.01 \mathrm{~mm}$ thick foil) was used to absorb most of the $\mathrm{Mn} \mathrm{K}_{\beta}$ line. The detector was filled with research-grade high-purity xenon gas, which is continuously purified as it circulates by thermal convection through getters (S.A.E.S. ST707).

\subsection{Different operation modes}

As the method relies on the absolute measurement of the ionization charge without any multiplication, the use of high counting rates is required to achieve a total charge $Q$, well within the sensitivity of the measuring electrometer. A high-enough electric charge (about $0.2 \mathrm{nC}$ corresponding to $\sim 5 \times 10^{6}$ photons dissipating each $5.9 \mathrm{keV}$ in the xenon gas) has to be collected in grid G1 during a period of time short enough to minimize losses caused by leakage currents, typically $10 \mathrm{~min}$ for our experimental setup. This implies the use of high counting rates, which unfortunately degrade the measurements of $n$ (in the second stage of the method), as a result of counting inefficiencies due to pulse pile-up. A compromise counting rate was found to be, for the present experiments, from about $5 \times 10^{3}$ to $10^{4}$ count $\mathrm{s}^{-1}$.

The charge-drift voltage characteristics of the ionization chamber were tested with the aim of finding the operational voltage plateau for the complete collection of the primary ionization charge $Q$. In Fig. 2, the integrated charge measured is shown as a function of the drift voltage $\Delta V$. At the high counting rates used, the drift voltage necessary to suppress charge recombination was found to be about $2000 \mathrm{~V}$ in xenon at 825 Torr. Above $\sim 2750 \mathrm{~V}$, the charge collected increases possibly as a result of interactions of photons (from scintillation) on other parts of the detector or on the electrodes surfaces. During these measurements the wire potential $V$ was about one-third of the drift voltage $\Delta V$, investigated to be moderately repulsive so that the collection of the primary electrons by the wire is inhibited. The effect of this wire potential 


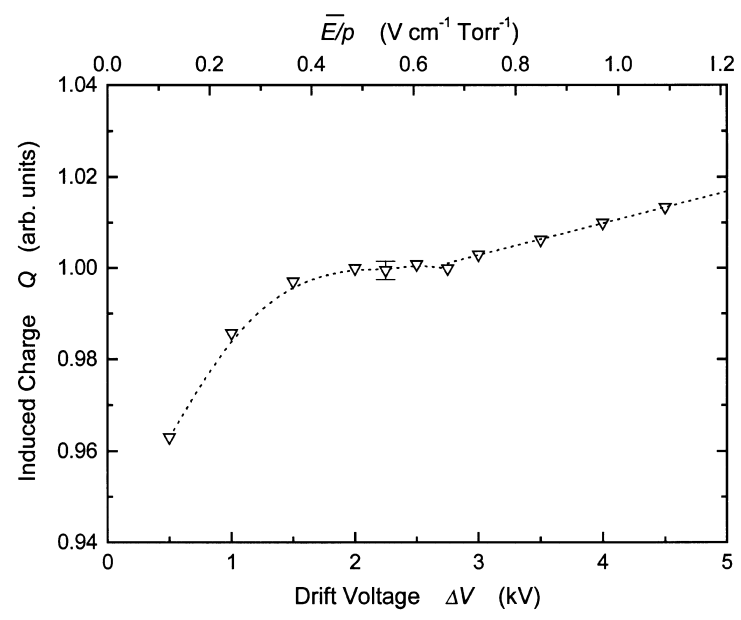

Fig. 2. Collected primary ionization charge $Q$ as a function of the drift voltage $\Delta V$, for a counting rate of $\sim 1.2 \times 10^{4}$ count $\mathrm{s}^{-1}$. The reduced electric field shown is averaged throughout the drift region.

$V$ in both the IC and the GPSC operation modes was experimentally studied with the aim of finding the best working conditions and is described in detail elsewhere [18].

The residual leakage currents in the IC were reduced to about $6 \mathrm{fA}$, including the electrometer offset current. To reach such low current levels, a guard ring was placed between grids $\mathrm{G} 1$ and $\mathrm{G} 2$ (Fig. 1), the detector-to-electrometer connection was electrostatically shielded and external interferences were minimized during operation.

The potential of having the three operation modes in the same hybrid detector affects significantly the PC geometry. In the PC mode, the distance from the anode to the cathode in the lower part of the detector ( $2 \mathrm{~cm}$ to $\mathrm{G} 1)$ is smaller than in its upper part ( $4 \mathrm{~cm}$ to radiation entrance window) (Fig. 1). Hence, the electrodes potentials had to be chosen appropriately to supply a symmetric gain in both directions for best PC performance [18]. For the PC pulses, the energy resolution obtained was $14.1 \%$, for counting rates of about $10^{2}$ count $\mathrm{s}^{-1}$, increasing to $16.8 \%$ for counting rates as high as $10^{4}$ count $\mathrm{s}^{-1}$, for $5.9 \mathrm{keV} \mathrm{X}$-rays in Xe at 825 Torr (Fig. 3).

The energy resolution achieved for the GPSC has not suffered deterioration in comparison to standard GPSCs energy resolution [17]. For $5.9 \mathrm{keV} \mathrm{X}$-ray photons in pure $\mathrm{Xe}$ at $825 \mathrm{Torr}$,

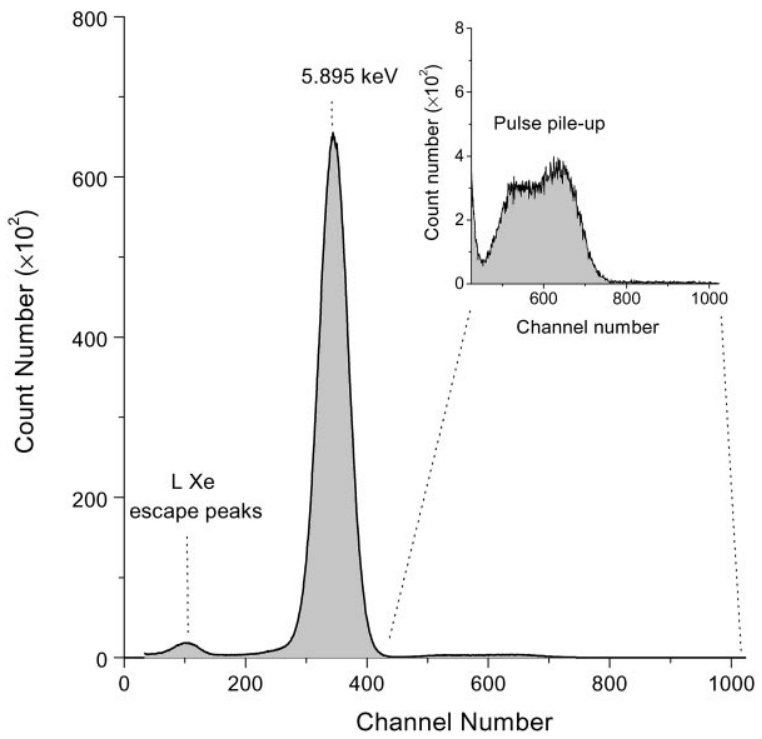

Fig. 3. Pulse-height distribution obtained with the PC for the $\mathrm{K}_{\alpha}$ line of $\mathrm{Mn}$, with a counting rate of $\sim 10^{4}$ count $\mathrm{s}^{-1}$.

the energy resolution obtained in the GPSC mode was $7.8 \%$ for low counting rates $\left(10^{2}\right.$ count $\left.\mathrm{s}^{-1}\right)$ and $9.0 \%$ for counting rates of about $5 \times 10^{3}$ count $\mathrm{s}^{-1}$ (Fig. 4).

The GPSC and PC pulses are fed to Camberra preamplifiers (PA), models 2005 and 2006, respectively. The PA pulses are then shaped and amplified with a Hewlett-Packard main amplifier, model HP5582A. For the high counting rates necessary in the $W$-value measurements, we used rather short shaping time-constants in the main amplifier with lower limits determined by the signal-to-noise ratio and the ballistic deficit. By shortening the pulse duration, the number of piled-up pulses decreases. Both integration and differentiation time constants were set equal to $0.5 \mu \mathrm{s}$. On the other hand, for the low counting rate measurements they were set equal to $5 \mu \mathrm{s}$.

The number $n$ of interactions in the gas is determined by selecting all the main peak events with a pulse discriminator. A high-speed EC performs the counting of these events.

\subsection{Corrections}

Two corrections were made to the $W$-value measurements to account for the escaped $\mathrm{L}$ xenon 


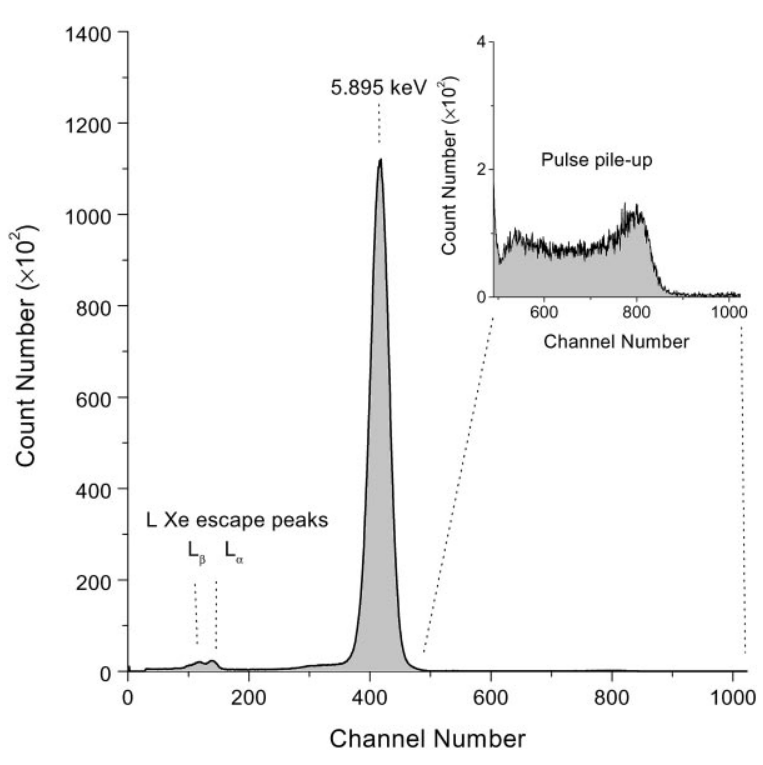

Fig. 4. Pulse-height distribution obtained with the GPSC for the $\mathrm{K}_{\alpha}$ line of $\mathrm{Mn}$, with a counting rate of $\sim 5 \times 10^{3}$ count s $^{-1}$.

fluorescence radiation and counting inefficiencies due to pulse pile-up. The pulse-height distributions were used to monitor the photon detectors operation.

\subsubsection{Escaped energy}

Some of the X-ray photons absorbed do not deposit their full energy in the gas, due to the escape of the fluorescence radiation from the detector active volume. The fluorescence photons are a product of the cascade decay of the vacancies in a photoionized xenon atom that follows the photoelectric effect absorption. The fluorescence yield in xenon is about $9 \%$ for the $\mathrm{L}$ shell photoionization. The amount of these fluorescence photons detected depends on the X-ray photon energy, the nature and pressure of the filling gas and the detector geometry and active volume.

In addition to $n$ full-absorption events, also $n_{\mathrm{e}}$ escape events contribute to the charge measured though with a lower weight. If each of these $n_{\mathrm{e}}$ escape events deposits an amount of energy $E_{\mathrm{d}}$ in the gas, the effective $W$-value is given by

$W=\left(n E+n_{\mathrm{e}} E_{\mathrm{d}}\right) /(Q / e)$

assuming that the $W$-value is the same for $E$ and $E_{\mathrm{d}}$. The ratio $n_{\mathrm{e}} / n$ for xenon was measured at low counting rates, using the corresponding peak areas from the MCA spectra obtained with both PC and GPSC photon detectors and was found to be equal to $3.6 \%$. For each counting rate used, $n_{\mathrm{e}}$ can be therewith determined. To obtain the deposited energy $E_{\mathrm{d}}$, the corresponding fluorescence emissions from the $\mathrm{L}_{1}, \mathrm{~L}_{2}$ and $\mathrm{L}_{3}$ xenon sub-shells were weighted by their fluorescence yields and ionization probabilities.

\subsubsection{Counting inefficiencies due to pulse pile-up}

Pile-up pulses can lead to events that cannot be resolved separately by the counting system. If not taken into account, the counting system dead time introduces a systematic error in the $W$-value measurements. These counting corrections were determined by applying the paralyzable model of dead time behavior to the counting rate measured [1]. This problem, which includes the choice of suitable dead time behavior of our counting system and the experimental dead time measurement, was studied [18] and will be reported soon. The measured dead time for xenon with the PC was $4.3 \mu$ s and with the GPSC was $3.9 \mu$ s. The counting rate corrections due to dead time were at most $4.7 \%$ of the counting rate measured.

\subsection{W-value for pure xenon}

For pure xenon at 825 Torr, the absolute $W$-value measured was $21.61 \mathrm{eV}$ with a statistical standard deviation of $0.04 \mathrm{eV}$ for a set of 12 separate measurements at different counting rates. When the other error sources are included (see Section 3.4), we obtain a $W$-value for xenon of $21.61_{-0.19}^{+0.28} \mathrm{eV}$, within a confidence level of approximately $95 \%$. The $W$-value obtained is in close agreement with other authors published results for X-rays for pure xenon, $21.5 \pm 0.4 \mathrm{eV}$ for energies in the $1.5-10 \mathrm{keV}$ range [9] and $21.7 \pm 0.5 \mathrm{eV}$ for $5.9 \mathrm{keV}$ [16]. The measured $W$-value is slightly lower than the one calculated by Monte Carlo simulation, $22.4 \mathrm{eV}$ [14] in our group, in Coimbra. These differences are being discussed.

\subsection{Error discussion}

The statistical standard deviation from the obtained distribution of $W$-value measurements is 
$0.04 \mathrm{eV}$. It corresponds to a standard deviation of $0.18 \%\left(u_{\mathrm{s}}\right)$. Residual leakage currents and charges, capacity or voltage fluctuations, electrometer offset current, background radiation, etc. were corrected by subtraction of the background measurements from the measurements with the X-ray source. The overall uncertainty of the results has the contributions described in the following sections.

\subsubsection{Electrometer $\left(u_{Q}\right)$}

The electrometer contributes to the ionization charge measurements with a standard uncertainty of $0.26 \%\left(u_{Q}\right)$, according to the device specifications.

\subsubsection{Counting rate measurement $\left(u_{n}\right)$}

The measured counting rate standard uncertainty is $0.30 \%\left(u_{n}\right)$, assumed to follow a normal distribution. The accuracy of counting rate measurements is limited by the counting inefficiency corrections. The counting rates measured with the PC and with the GPSC agree within this uncertainty.

\subsubsection{L fluorescence escape events $\left(u_{\mathrm{e}}\right.$ and $\left.u_{W \mathrm{e}}\right)$}

The ratio of the areas of the L-escape and full absorption peaks (used as a measure of the ratio $n_{\mathrm{e}} / n$ ) has a $12 \%$ standard deviation, assuming a normal distribution. On the measured $W$-value this factor contributes with a $\pm 0.10 \%$ standard uncertainty $\left(u_{\mathrm{e}}\right)$. On the other hand, the correction used assumes that the $W$-value for the energy absorbed in the gas $E_{\mathrm{d}}$ in the case of escape events is the same as for the main energy peak $E$. However, variations of the $W$-value with the X-ray energy are well known $[14,15]$. The $W$-value for Xe L fluorescence photons is about $0.35 \mathrm{eV}$ lower than the $W$-value for $5.9 \mathrm{keV} X$-ray photons $[14,15]$. Therefore, the $W$-value of an escape event is higher than for the case in which the fluorescence photon is absorbed in the gas, and was then underestimated in the correction of Eq. (3). This factor determines a maximum uncertainty contribution of $+0.06 \%$ $\left(u_{W \mathrm{e}}\right)$ in the $W$-value measurements.

\subsubsection{Remaining $M n K_{\beta}$ photons $\left(u_{\mathrm{K} \beta}\right)$}

The chromium foil ( $0.01 \mathrm{~mm}$ thick), used between the radioactive source and the detector, absorbs about $95 \%$ and $38 \%$ of the $\mathrm{Mn} \mathrm{K}_{\beta}$ and $\mathrm{Mn}$
$\mathrm{K}_{\alpha}$ lines, respectively. Thus, as the $\mathrm{Mn}$ fluorescence $\mathrm{K}_{\beta}$ yield is about $12 \%$ of the $\mathrm{K}_{\alpha}$ yield, only about $1 \%$ of the photons interacting in the gas originate from the $\mathrm{Mn} \mathrm{K}_{\beta}$ line. The $W$-value for these $6.5 \mathrm{keV}$ photons is very near (about $0.5 \%$ lower) the value for $5.9 \mathrm{keV}$ events according to the Monte Carlo simulation results [14], as there are no sudden changes in photoionization cross sections between these two energies. Therewith these events contribute to an uncertainty in the $W$-value of $+0.01 \%\left(u_{\mathrm{K} \beta}\right)$.

\subsubsection{Photoelectron ejection from the radiation window $\left(u_{\mathrm{ph}}\right)$}

The possibility of photoelectric effect in the Kapton window or the aluminum film covering it can be a source of a systematic error. An upper limit for the error due to the electrons ejected from the radiation entrance window was estimated assuming that for all the X-ray interactions at a distance smaller than $R_{\mathrm{e}} \sim 380 \mathrm{~nm}$ (the photoelectron range in the double layer of Kapton and aluminum) from the window surface, half of the electrons are ejected to the gas. Using cross-section data [19], the ratio of events absorbed in this $R_{\mathrm{e}}$ thick double layer and absorbed in xenon leads to an evaluated maximum uncertainty contribution of $+0.24 \%\left(u_{\mathrm{ph}}\right)$ in the $W$-value measured. This error can be reduced in a future prototype by the use of beryllium windows.

\subsubsection{Impurities}

The role of impurities in our system can be of great importance in these measurements on account of Penning effects [20], which increase the ionization yield, therefore reducing the $W$-value measured. This was tested by switching off the heating responsible for the gas continuous purification with getters. The results in Fig. 5 indicate that such effects appear not to affect significantly the $W$-value measurements at the low residual level of impurities existing in the system. The observed slight increment in the $W$-value is probably due to the existence of atomic and molecular impurities, which by collisions with primary electrons, atomic or molecular excitation or even molecular cracking, increase the amount of energy spent in non-ionizing inelastic collisions.

The electronegative impurity effects in the gaseous ionization detectors are one of the main causes 


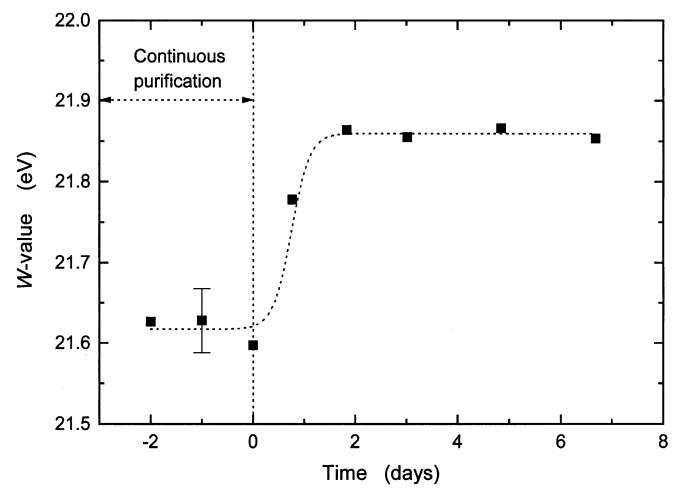

Fig. 5. Impurity effects on the measurements: $W$-values measured as a function of time before and after switching off the purification system heating power supply. The standard deviation shown is due only to statistical factors.

of pulse amplitude reduction due to primary electron attachment (leading also to energy resolution deterioration). Our technique, being a DC one, based on the collection of both electrons and negative ions (besides the positive ions), is therefore little affected by electronegative impurities, in opposition to other techniques. The main effect of these impurities on the PC and GPSC operation modes is the energy resolution deterioration, which does not affect the counting rate determination within the impurity levels involved in our system. The effect of impurities was therefore considered negligible.

\subsubsection{Photoemission from electrodes surfaces $\left(u_{\mathrm{pe}}\right)$}

During the charge measurement step, the primary scintillation can lead to photoemission from the surrounding electrodes. The number of primary scintillation photons, indicated by Monte Carlo simulation, is approximately $65 \%$ of the number of primary electrons [21]. However, since the quantum yield of the stainless steel surrounding surfaces, for the peak of the xenon emission spectra $(\sim 171 \mathrm{~nm})$ [22], is below $10^{-3}$ [23], the reduction in the $W$-value due to this factor is under $+0.07 \%$ $\left(u_{\mathrm{pe}}\right)$. Although we have no data for the quantum yield of $\mathrm{Al}$ (which is deposited on the radiation window), as the surrounding average solid angle of $\mathrm{Al}$ surface is between $10 \%$ and $20 \%$, its contribu- tion should be even lower and was considered negligible.

\subsubsection{Summary}

The error sources, using the terminology of NIST [24], which lead to the uncertainty of our measurements, include error sources evaluated by statistical methods whose standard uncertainties are $u_{\mathrm{s}}, u_{Q}, u_{n}$ and $u_{\mathrm{e}}$, as well as contributions due to systematic errors whose uncertainties $u_{W \mathrm{e}}$, $u_{\mathrm{K} \beta}, u_{\mathrm{ph}}$ and $u_{\mathrm{pe}}$ are estimated by other means than experimental measurements. A standard uncertainty $u$ of $0.45 \%$ due to the statistical error sources was determined by quadratically adding the standard uncertainties, assuming normal (Gaussian) and independent distributions, corresponding to a $68 \%$ confidence interval:

$u=\left(u_{\mathrm{s}}^{2}+u_{Q}^{2}+u_{n}^{2}+u_{\mathrm{e}}^{2}\right)^{1 / 2}$.

For a confidence level of approximately $95 \%$, the expanded uncertainty due to these error sources is thus $2 u$.

As $u_{W \mathrm{e}}, u_{\mathrm{K} \beta}, u_{\mathrm{ph}}$ and $u_{\mathrm{pe}}$ are upper limits of systematic errors, we calculated a maximum uncertainty of $+0.38 \%\left(u_{W \mathrm{e}}+u_{\mathrm{K} \beta}+u_{\mathrm{ph}}+u_{\mathrm{pe}}\right)$ due to these errors. The combined expanded uncertainty $U$ was then obtained from the expressions

$U=+2 u+u_{W \mathrm{e}}+u_{\mathrm{K} \beta}+u_{\mathrm{ph}}+u_{\mathrm{pe}}$

for the upper limit, or

$U=-2 u$

for the lower limit, leading to values of $+1.27 \%$ and $-0.89 \%$. For a confidence level of approximately $95 \% U$ is thus lower than $\pm 1.27 \%$. Assuming half Gaussian distributions for the uncertainties of the systematic errors, the standard uncertainty is $+0.64 \%$ and $-0.45 \%$.

\section{Conclusions}

We have presented a new technique for the absolute measurement of $W$-values for a wide range of gases at atmospheric pressures and low-energy Xrays. It was shown that accuracies better than the ones described before in the literature are achievable. For xenon at standard pressures, the $W$-value 
obtained was $21.61_{-0.10}^{+0.14} \mathrm{eV}$, within a confidence level of $68 \%$ or $21.61_{-0.19}^{+0.28} \mathrm{eV}$, within a confidence level of $95 \%$. Such accuracies are required to confirm work that predicts variations of the $W$-values with the photon energy [14]. Since the only experimental $W$-value published results concerning the dependence on the energy are relative ones [15], the development of an absolute measurement technique such as the present one is important. Other applications of this technique include the study of pure gases, or their mixtures, without a quenching gas, which are difficult to study with most other techniques.

A new design is being planned that includes only a PC in the second stage, with the aim of measuring $W$-values at high pressures, where different collisional processes may be involved in primary electron production, thus leading to different $W$-values. This was an additional limitation of the previous technique [16] due to the inclusion of a GPSC with a PMT, which limited the maximum pressure allowed.

\section{Acknowledgements}

This work was carried out in the Atomic and Nuclear Instrumentation Group of the Instrumentation Center (Unit 217/94) of the Physics Department, University of Coimbra. It was supported by project Praxis/3/3.1/CTAE/1920/95. F.L.R.Vinagre was supported by the PRAXIS XXI/BM/6551/95 research grant. We thank our colleagues in the Group, especially Dr. Teresa H.V.T. Dias for helpful discussions.

\section{References}

[1] G.F. Knoll, Radiation detection and measurements, 2nd Edition, Wiley, New York, 1989.

[2] W.P. Jesse, Phys. Rev. 174 (1968) 174.
[3] S. Kubota, J. Phys. Soc. Jpn. 29 (1970) 1017.

[4] N. Ishida, J. Kikuchi, T. Doke, Jpn. J. Appl. Phys. 31 (1992) 1465.

[5] T. Takahashi, S. Konno, T. Hamada, M. Miyajima, S. Kubota, A. Nkamoto, A. Hitashi, E. Shibamura, T. Doke, Phys. Rev. A 12 (5) (1975) 1771.

[6] M. Miyajima, T. Takahashi, S. Konno, T. Hamada, S. Kubota, A. Nkamoto, E. Shibamura, T. Doke, Phys. Rev. A 9 (3) (1974) 1438.

[7] M. Järvinen, H. Sipilä, Nucl. Instr. and Meth. 217 (1983) 282.

[8] M. Järvinen, H. Sipilä, Nucl. Instr. and Meth. 193 (1982) 53.

[9] P.B. Lyons, J.A. Baran, J.H. McCrary, Nucl. Instr. and Meth. 95 (1971) 571.

[10] D. Srdoc, Nucl. Instr. and Meth. 108 (1973) 327.

[11] I. Bronić, B. Grosswendt, Nucl. Instr. and Meth. B 117 (1996) 5.

[12] A. Pansky, A. Breskin, R. Chechik, J. Appl. Phys. 79 (12) (1996) 8892.

[13] A. Pansky, A. Breskin, R. Chechik, J. Appl. Phys. 82 (2) (1997) 871.

[14] T.H.V.T. Dias, F.P. Santos, A.D. Stauffer, C.A.N. Conde, Phys. Rev. A 48 (4) (1993) 2887.

[15] C. Budtz-Jørgensen, C. Olesen, H.W. Schnopper, T. Lederer, F. Scholze, G. Ulm, Nucl. Instr. and Meth. A 367 (1995) 83.

[16] F.I.G.M. Borges, C.A.N. Conde, Nucl. Instr. and Meth. A 381 (1996) 91.

[17] J.M.F. Dos Santos, A.C.S.S. Bento, C.A.N. Conde, X-ray Spectrom. 22 (1993) 328.

[18] F.L.R. Vinagre, Technique for the W-value absolute measurement for X-rays in gases: results in $\mathrm{Ne}-\mathrm{Xe}$ mixtures, M.Sc. Degree Thesis, Physics Department, University of Coimbra, Portugal, 1999.

[19] J.W. Robinson, Handbook of Spectroscopy, Vol. 1, 2nd Edition. CRC Press Inc, Boca Raton, 1979.

[20] C.E. Melton, G.S. Hurst, T.E. Bortner, Phys. Rev. 96 (3) (1954) 643.

[21] F.P. Santos et al., private communication.

[22] T. Takahashi, S. Himi, M. Susuki, J. Ruan(Gen), S. Kubota, Nucl. Instr. and Meth. A 205 (1983) 591.

[23] J. Séguinot, G. Charpak, Y. Giomataris, V. Peskov, J. Tischhauser, T. Ypsilantis, Nucl. Instr. and Meth. A 297 (1990) 133.

[24] B.N. Taylor, C.E. Kuyatt, Guidelines for evaluating and expressing the uncertainty of NIST measurements results, NIST Technical Note 1297, National Institute of Standards and Technology, 1994 Edition. 